\title{
La Aplicación de Registros de Control y la Eficiencia Administrativa en las Pequeñas Empresas de Manabí
}

\section{The Application of Control Registers and Administrative Efficiency in the Small Companies of Manabí}

\section{A Aplicação de Registros de Controle e Eficiência Administrativa nas Pequenas Empresas de Manabi}

Miguel A. Baque-Cantos I

miguel.baque@unesum.edu.ec

Arturo A. Álvarez-Indacochea ${ }^{\text {II }}$

arturo.alvarez@unesum.edu.ec

\author{
Narda P. Merchán-Ponce ${ }^{\text {III }}$ \\ narda.merchan@unesum.edu.ec \\ Adela del Jesús Lucio-Pillasagua ${ }^{\text {IV }}$ \\ adela.lucio@unesum.edu.ec
}

Recibido: 27 de junio de 2018 * Corregido: 28 de agosto de 2018 * Aceptado: 15 de septiembre de 2018

I. Ingeniero Comercial, Diplomado en Autoevaluación y Acreditación, Magister, Docencia Universitaria e Investigación Educativa, Docente de la Universidad Estatal del Sur de Manabí, Jipijapa, Ecuador.

II. Ingeniero Comercial, Magister, Gerencia Educativa, Docente de la Universidad Estatal del Sur de Manabí, Jipijapa, Ecuador.

III. Economista, Master en Administración de Empresas, Docente de la Universidad Estatal del Sur de Manabí, Jipijapa, Ecuador.

IV. Ingeniera en Auditoria, Magister en Gerencia Educativa, Docente de la Universidad Estatal del Sur de Manabí, Jipijapa, Ecuador. 


\section{Resumen}

El presente trabajo sintetiza la investigación de La Aplicación de Registros de Control y la Eficiencia Administrativa en las pequeñas empresas de la provincia de Manabi y la integración de los procesos de planificación que son los encargados en cumplir con los objetivos generales y específicos de toda empresa. El conocimiento de modelos de registros internos establecidos en otras empresas y el proceso de cómo aplicarlos a las pequeñas empresas de la provincia con el fin de generar mayores beneficios que impulsen el desarrollo empresarial, así como también satisfacer las necesidades de los consumidores, bajando los costos de producción y manteniendo la calidad de los productos.

Los sistemas de control planteados son el modelo COSO que es uno de los más antiguos y el sistema $\mathrm{ABC}$ ambos se basan en la realización de encuestas y cuestionarios que son analizados e interpretados por cada jefe de departamento parta finalmente brindar soluciones que impulsen la eficiencia administrativa en las pequeñas empresa de la provincia. En el estudio se observó que a pesar de que existen varios sistemas de control la mayoría se rige por el modelo COSO, que la finalidad de los mismos es implementar un conjunto de reglas por departamento que a su vez están interrelacionadas y que contribuyen en el manejo adecuado de los recursos financieros de toda organización.

Palabras clave: Registro, Control, Eficiencia, Empresa, Organización. 


\begin{abstract}
The present work notetizó the investigation of the Application of Control Registers and the Administrative Efficiency in the small companies of the province of Manabi and the integration of the processes of planning that are the attendants to fulfill with the general and specific objectives of all the company. Knowledge of models of internal records established in other companies and the process of how to apply the small companies of the province in order to generate greater benefits that boost the development of the company, as well as satisfy the needs of consumers, lower the production costs and the quality of the products.

The control systems are designed for the model that is based on conducting surveys and questionnaires that are analyzed and interpreted by each department head who finally receive solutions that boost administrative efficiency, the small companies of the province. In the study there are several models that control the majority of the COSO model, which is responsible for the system being in place and doing so in the proper management of the financial resources of any organization.
\end{abstract}

Keywords: Registration, Control, Efficiency, Company, Organization. 


\section{Introducción.}

No es un secreto para nadie que el objetivo de cualquier empresa sin importar el sector o tamaño de la misma es generar beneficios económicos y/o financieros que los impulsen a tener una mejor rentabilidad tanto en sus procesos productivos como generar valor a los inversionistas, además de brindar seguridad a sus trabajadores. La rentabilidad de una empresa se verá afectada según sea la manera en que se llevan los registros y sistemas de control, ya que, de esta forma, sin importar la complejidad del proceso se garantiza una gestión adecuada que permitan optimizar la competitividad de la empresa en el mercado.

Hoy en día con el desarrollo exponencial y sustentado de las grandes corporaciones, es imperioso para la pequeña y mediana empresa el desarrollo y acatamiento de planes y métodos de control que le permitan mantenerse a la par con las exigencias del mercado que cada vez son mayores tanto de calidad como en tiempos de respuesta.

Cada empresa tiene sus propios objetivos operacionales muy diferenciados a otras empresas, ya que los mismos están relacionados con las prioridades, el juicio y el estilo de gestión de la gerencia. Es fundamental que este tipo de objetivos estén claramente definidos y coordinados con los demás objetivos y estrategias específicos, ya que, es sobre los objetivos operativos hacia donde la empresa dirige una parte sustancial de sus recursos. (de Jaime Eslava, 2013, p.14).

Los registros de control en las empresas no son más que estrategias que deben implantarse con el fin de cumplir los objetivos generales y especificos. Los dueños y gerentes se ven en la obligacion de crear y fomentar medidas que impulsen las mejoras y mantengan el control dentro de toda organización siendo pieza fundamental las metas establecidas bien sea en pequeñas, medianas y

\footnotetext{
117 Vol. 4, núm. 4, octubre 2018, pp. 114-127 Miguel A. Baque-Cantos; Arturo A. Álvarez-Indacochea; Narda P. Merchán-Ponce; Adela del Jesús Lucio-Pillasagua
} 
grandes empresas. Las estrategias buscan formalizar los procesos de producción y de planificación para mantener un registro en el control de todas sus operaciones.

La eficiencia en la adminitracion de los recursos empresariales es un reflejo del control existente en la operatividad y los procesos, al tener una adecuada administracion del capital se logra cumplir con los objetivos que estan planteados por toda empresa. En las pequeñas empresas al igual que en las grande y medianas es de gran importancia implantar estrategias de control que aseguren la eficiencia administrativa de los recursos y que brinden seguridad a sus trabajadores.

Las empresas pequeñas deben reinventar su modelo de negocio basadas en una fuerza productiva que contemple una estructura corporativa y competitiva centrada en la cultura de la planificación, el talento humano calificado, la flexibilidad y adaptabilidad del negocio, el marketing, las tecnologías de punta, la asociatividad empresarial y la estimulación del pensamiento estratégico de sus gerentes para que puedan sostener sus ventajas empresariales girando en torno a la frontera de la competitividad. (Parra, 2014, p.134).

La idea de la existencia de los registrso de control en toda empresa, está basada en mantener un orden en la planificacion y los procesos empresariales donde se refleje la eficiencia administrativa a traves del manejo adecuado de los recursos y el capital, y como idea principal impulsar al crecimiento de la pequeña empresa y superar sus propios expectativas cada año.

Manabi es una de las 24 provincias del Ecuador situada en la zona costera las acitividades principales de la provincia son el comercio, la pesca, la ganaderia y la industria, es la tercera provincia mas poblada del pais, por lo que la formacion de pequeñas empresas es bastante debido a que se tiene mucho campo de trabajo y produccion. Visto esto es importante por parte de las

\footnotetext{
118 Vol. 4, núm. 4, octubre 2018, pp. 114-127 Miguel A. Baque-Cantos; Arturo A. Álvarez-Indacochea; Narda P. Merchán-Ponce; Adela del Jesús Lucio-Pillasagua
} 
organizaciones mantener estrategias que controlen los procesos e impulsen la eficiencia administrativas para las empresas de la zona.

Por lo tanto, los registros de control en la de planificacion para las pequeñas empresas de la provincia de Manabi garantizan el logro de los objetivos especificos en las pequeñas empresas de la zona y el impulso de la region en general, logrando llegar más allá de sus propias fronteras incrementando las tasas de empleo y al desarrollo del país.

\section{Método y Materiales.}

Esta investigación está enfocada en el estudio de la aplicación de registros de control, la eficiencia administrativa en las pequeñas empresas de la provincia de Manabi y la integración de los procesos y la planificación que hacen posible el logro de los objetivos generales y específicos de toda organización, a través de la recopilación de información importante y de estudios de modelos de registros de control internos aplicados en diferentes empresas que han proporcionado eficiencia en el manejo de los recursos y el capital financiero, en busca de lograr bajar los costos de producción, manteniendo la calidad de los productos y cumpliendo con las exigencias de los consumidores.

La revisión se ha centrado en textos, documentos y artículos científicos publicados disponibles en la web, considerando que aquella herencia de la globalización nos permite acceder a mayor y mejor información a través de las herramientas tecnológicas. El motor de búsqueda ha sido herramientas académicas de la web que direccionan específicamente a archivos con validez y reconocimiento científico, descartando toda información no confirmada o sin las respectivas referencias bibliográficas.

\footnotetext{
119 Vol. 4, núm. 4, octubre 2018, pp. 114-127 Miguel A. Baque-Cantos; Arturo A. Álvarez-Indacochea; Narda P. Merchán-Ponce; Adela del Jesús Lucio-Pillasagua
} 


\section{Resultados.}

La implementacion de los registros de control en las pequeñas empresas aseguran el cumplimiento de los objetivos especificos y generales, beneficiando los logros y garantizando una mejor gestión en la planificación de sus procesos. Las pequeñas empresas se ven en la obligación de crear estrategias donde se cordinen los registros de control necesarios para el cumplimiento de sus objetivos.

El marco conceptual de control interno o de registro de control empresarial más reconocido es el modelo COSO propuesto en 1992 por el Committe of Sponsoring Organizations of the Treadway Commission (COSO), (Parra, 2014, p.131). A continuación se presenta el modelo de control COSO

\section{Imagen $N^{o} 1$ Modelo de Control COSO}

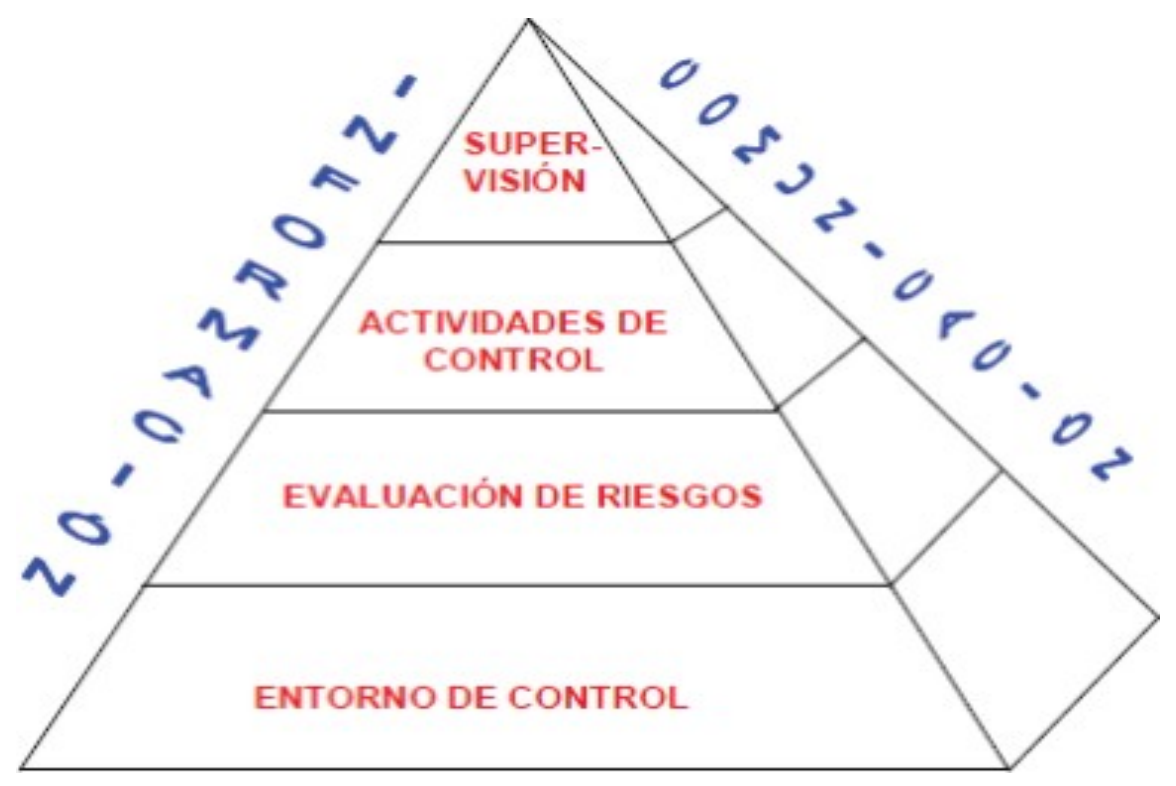

Fuente: (Maria Alvarado, 2011, p. 71). 
En la imagen $\mathrm{N}^{\mathrm{o}} 1$ se observa, el modelo de control COSO como un conjunto de normas que son las encargadas de la administración dentro de la organización, cada departamento es el responsable de hacer cumplir sus reglamentos dentro de la empresa y a la vez estos se fusionan en un solo modelo en que se integran todos los reglamentos para brindar seguridad sobre el logro de los objetivos específicos y generales de la entidad con relación a la confianza en las actividades financieras, la efectividad y eficiencia de las operaciones, y el cumplimiento de las leyes y regulaciones aplicables. (Maria Alvarado, 2011, p. 59).

Este modelo aplicado a pequeñas y medianas empresas brinda eficiencia en todo el ambito de planificación que engloba a la organización, el modelo busca integrar los procesos que se agrupan a nivel empresarial y mantener los registrso de control pertinentes por cada departamento. A continuacion se presenta un diseño metodológico en el que se aplica el Modelo COSO.

\footnotetext{
121 Vol. 4, núm. 4, octubre 2018, pp. 114-127 Miguel A. Baque-Cantos; Arturo A. Álvarez-Indacochea; Narda P. Merchán-Ponce; Adela del Jesús Lucio-Pillasagua
} 


\section{Imagen $N^{o}$ 2. Diseño Metodológico.}

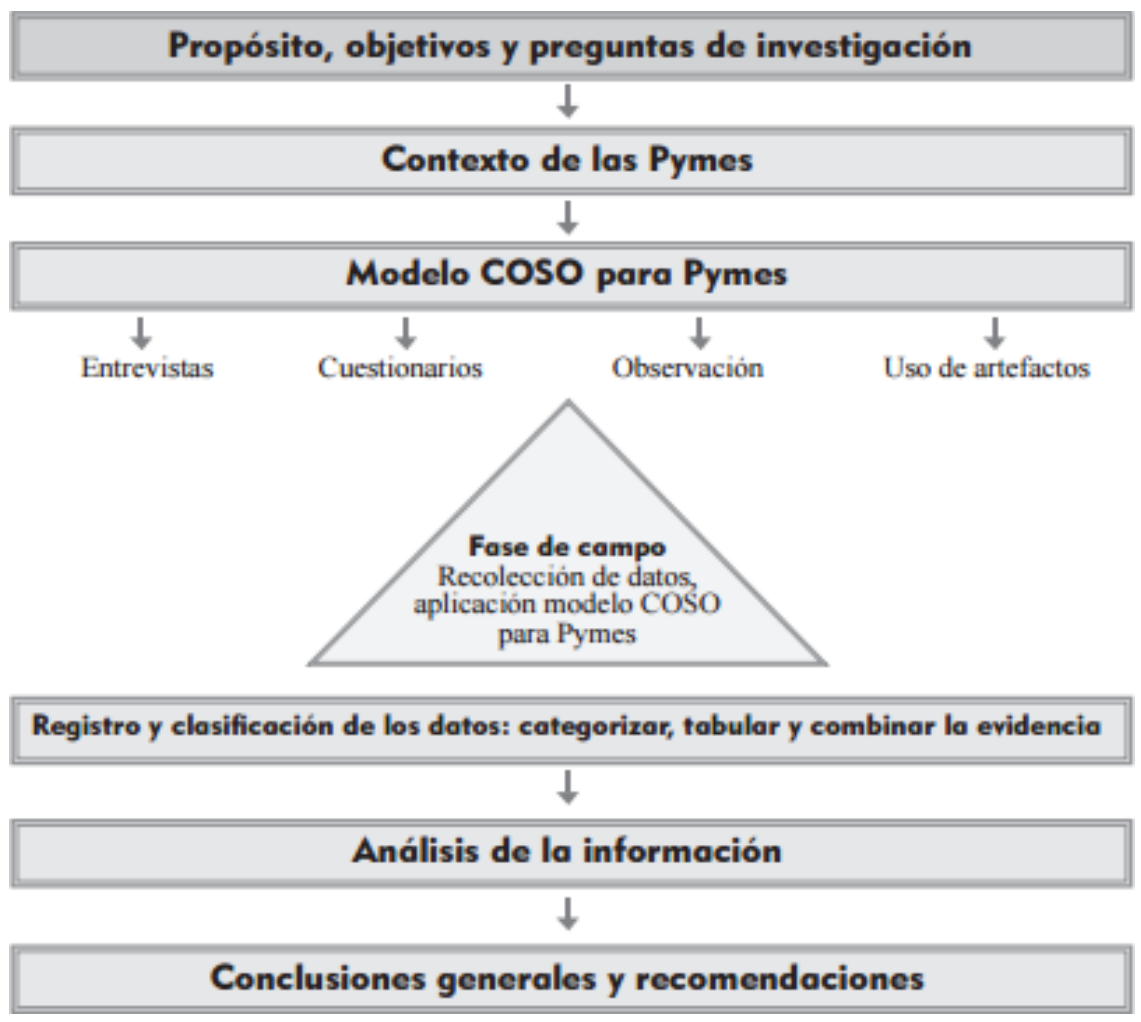

Fuente: (Parra, 2014, p. 4).

En la imagen $\mathrm{N}^{\mathrm{o}} 2$ se observa primero se debe tener definidos los objetivos y el proposito para formular las preguntas de investigación, conocer el contexto de la empresa para luego aplicar el modelo COSO que consiste en realizar una serie de entrevistas, cuestionarios y observaciones con el uso de herramientas necesarias para la implementación del modelo, una vez realizado ésto se procede a recolectar los datos y a aplicar el modelo comenzando por el registro, la selección de los datos obtenidos donde se busca clasificar, ordenar y relacionar las evidencias tomadas en los ensayos de campo para luego analizar la información y finalmente llegar a la conlusión y recomendaciones de las estrategias que se deben aplicar en los registros de control con la finalidad

\footnotetext{
122 Vol. 4, núm. 4, octubre 2018, pp. 114-127

Miguel A. Baque-Cantos; Arturo A. Álvarez-Indacochea; Narda P. Merchán-Ponce; Adela del Jesús Lucio-Pillasagua
} 
principal de impulsar la efíciencia de los procesos administrativos y lo relacionado a la planificación de las pequeñas empresas.

A pesar de la existencia de otros sistemas o estratégias aplicadas en el registro de control para empresas, el modelo COSO ha servido como patrón para los demás, un ejemplo de ésto es el sistema $\mathrm{ABC}$ o como sus siglas en inglés lo definen "Activity Based Costing" o "Costeo Basado en Actividades" que al igual que el modelo COSO consiste en un conjunto de actividades a seguir en donde se evalúan estratégias que son implementadas para lograr el desarrollo de los objetivos tanto generales como específicos de toda empresa.

La implementación de las bases del perfeccionamiento empresarial impone nuevos métodos de gestión que permitan obtener una mayor eficiencia mediante el uso racional de los recursos materiales, financieros y humanos, lo que lleva implícito la disminución sistemática de los costos de la producción de bienes y servicios. (Lara Zayas, 2007).

En la República del Ecuador se registraron alrededor 843.745 empresas para el año 2016. 745 empresas divididas en 19 actividades económicas como Comercio, Agricultura, Transporte, etc., según los últimos datos del Directorio de Empresas y Establecimientos 2016 publicado por el Instituto Nacional de Estadísticas y Censos (INEC). (INEC, 2017).

En la provincia de Manabi los principales sectores económicos son la agricultura, ganadería, silvicultura y pesca, explotación de minas y canteras, industrias manufactureras, comercio, construcción y servicios. A continuación se presenta una imagen en donde se observa los diferentes sectores económicos de las pequeñas y medianas empresas (PyME) de la provincia y sus respectivos porcentajes.

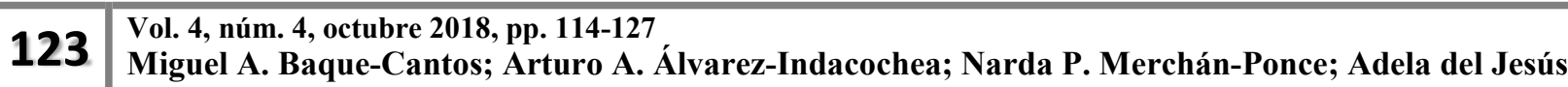
Lucio-Pillasagua
} 
Imagen No3. Principales sectores económicos de las Pequeñas y Medianas Empresas (PyME)

\section{ubicadas en la provincia de Manabí}

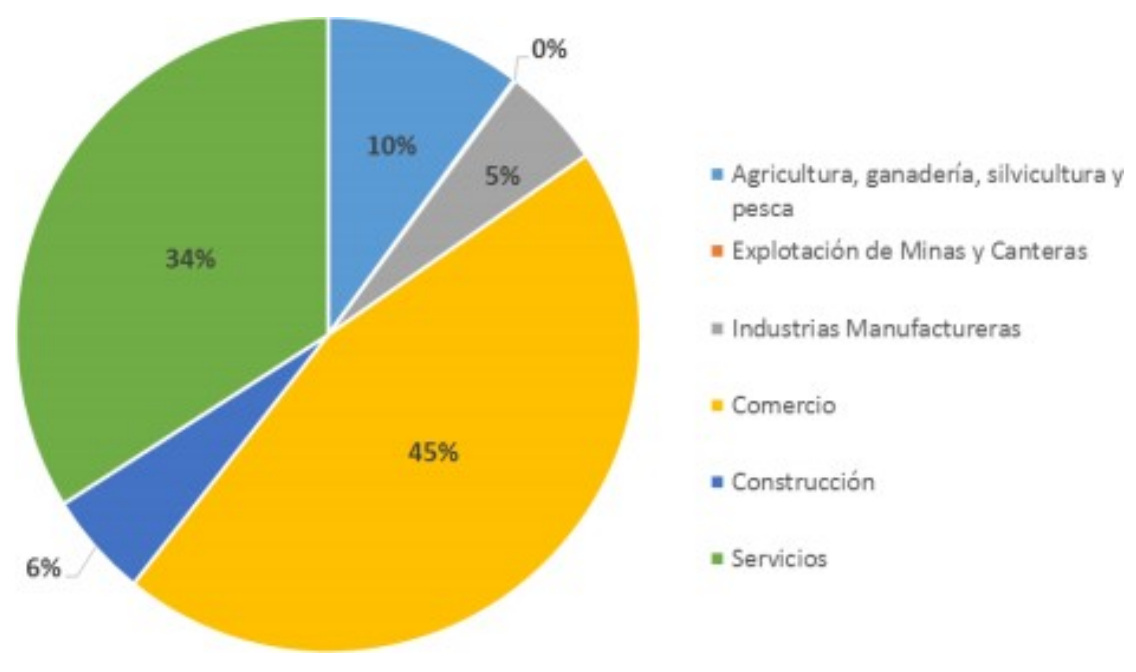

Fuente: (Universidad Andina Simón Bolívar, 2014, p.5).

Como se observa en el diagrama según el Directorio de Empresas 2014 del INEC, las PYME de Manabí se concentran principalmente en el sector de comercio (45\%). (Universidad Andina Simón Bolívar, 2014).

El Directorio de Empresas y Establecimientos recopila información a partir de registros administrativos generados por el Servicio de Rentas Internas (SRI), el Instituto Ecuatoriano de Seguridad Social (IESS) y el Ministerio de Educación (MINEDUC), brindando información sobre el total de unidades económicas, empleo y ventas desde una perspectiva sectorial y territorial. Según las cifras se tiene que las empresas pequeñas a nivel nacional registraron un 7,5\% de ventas anuales que representa entre 100.001 a 1'000.000 de dólares, con un numero de entre 10 y 49 empleados. (INEC, 2017)

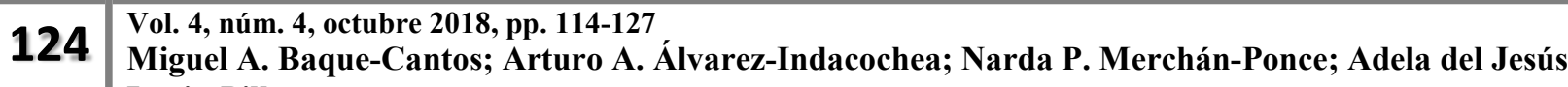
Lucio-Pillasagua
} 
Del 7,5\% de las ventas por pequeñas empresas registradas a nivel nacional el 3,3\% pertenece a la provincia de Manabi, se observa que casi la mitad del porcentaje de ventas se ubica en esta zona del pais, ésto debido a que la provincia representa la tercera más grande de la Republica del Ecuador y donde se concentra una parte significativa del comercio empresarial.

El uso de sistemas de registro de control en las empresas ubicadas en la provincia de Manabi ha brindado una mayor efeciciencia en la administracion de los recursos y en la planificacion de los procesos y como consecuencia un porcentaje bastante significativo en las ventas anuales registradas. A medida que se implementan reglas de control que mantienen la interrelacion entre los departamento empresariales se ha visto un aumento en las mejorías de las organizaciones. Toda empresa siempre busca aumentar la calidad de sus productos y bajar los costos existentes, es por esto que mantener registros de control en cada uno de los procesos por muy pequeñas o grandes que sean las empresas siempre brindará mejoras necesarias para lograr los objetivos generales y específicos.

Por lo tanto, en un analisis de los resultados y teniendo en cuenta la implementación de registros de control que obligan de forma sistematizada y organizada el desarrollo de los objetivos generales y específicos de las pequeñas empresas de Manabi, se observa que por ser la provincia una de las más grandes de la República del Ecuador además de estar ubicada en zona costera, el porcentaje de ventas es bastante significativo y su sector economico se concentra principalemente en el sector comercio.

\footnotetext{
125 Vol. 4, núm. 4, octubre 2018, pp. 114-127 Miguel A. Baque-Cantos; Arturo A. Álvarez-Indacochea; Narda P. Merchán-Ponce; Adela del Jesús Lucio-Pillasagua
} 


\section{Discusión y Conclusiones.}

Para lograr la eficiencia administrativa tanto en pequeña como en grandes empresas se ha observado que al implementar estratégias o reglas que controlen el uso de los recursos económicos así como tambien el manejo del capital se evidencian mejoras en los procesos y la planificación de los objetivos generales y específicos de toda organización.

En el estudio se planteo el uso del modelo COSO que consiste en un conjunto de reglas que se implementan por departamento y que a su vez se interrelacionan entre si, este modelo es el más usado y es tomado como patrón para los diferentes modelos de registro de control empresarial que existen.

Se observó que, en la provincia de Manabi las ventas anuales de pequeñas empresas representan el 3,3\% del porcentaje total a nivel nacional y que su principal sector económico se concentra en el comercio, ésto debido a que la provincia es la tercera más grande de la República y que esta ubicada en una zona costera, por lo que representa de gran importancia que los dueños de pequeñas empresas se encarguen de implementar sistemas de registros de control que impulsen los objetivos empresariales, de tal manera que exista un mayor compromiso en aumentar la producción bajando los costos y manteniendo la calidad de los productos.

Por tanto la rentabilidad de las pequeñas empresas de la provincia de Manabi se ve reflejada en el manejo de los registros de control, ya que, de esta manera se garantiza una gestion adecuada de los procesos permitiendo optimizar la competitividad de las PyME en los mercados nacionales y traspasar sus propias fronteras.

\footnotetext{
126 Vol. 4, núm. 4, octubre 2018, pp. 114-127 Miguel A. Baque-Cantos; Arturo A. Álvarez-Indacochea; Narda P. Merchán-Ponce; Adela del Jesús Lucio-Pillasagua
} 
Debido al crecimiento en las exigencias que se tienen hoy en día por parte de los consumidores es importante que las pequeñas empresas acaten planes y métodos de control que vaya de la mano con las exigencias del mercado. Por lo tanto, aquellas empresas que aún no aplican sistemas de registros, es recomendado tomar las medidas pertinentes e implantar modelos de control interno como el modelo COSO o el $\mathrm{ABC}$ que le ayuden a reorganizar sus pequeñas empresas y asegurar el cumplimiento de los objetivos para los cuales se han creado, ya que, en ocasiones sucede que hay empresas que desvían este tema por falta de control ocasionando pérdidas que podrían corregirse si se les presta mayor atención a los procesos de planificación y producción integrandolos como un conjunto de actividades que dan por sentado la eficiencia en la administración de los recursos y finanzas de toda organización.

\section{Bibliografia.}

Alvarado Veletanga, M., \& Tuquiñahui Paute, S. (2011). Propuesta de implementación de un sistema de control interno basado en el modelo COSO, aplicado a la empresa Electro Instalaciones en la ciudad de Cuenca. Quito: UPS.

Castañeda Parra, L. (2014). Los sistemas de control interno en las Mipymes y su impacto en la efectividad empresarial. Revista En-contexto, 2(2), 129-146.

INEC. (2017). Ecuador registró 843.745 empresas en 2016. Quito: Instituto Nacional de Estadística y Censos.

Lara Zayas, M. (2007). Cálculo de los costos de servicios internos en una empresa comercial. Obtenido de https://www.gestiopolis.com/calculo-de-los-costos-de-servicios-internos-enuna-empresa-comercial/

Universidad Andina Simón Bolívar, S. E. (2014). Observatorio de la PyME.

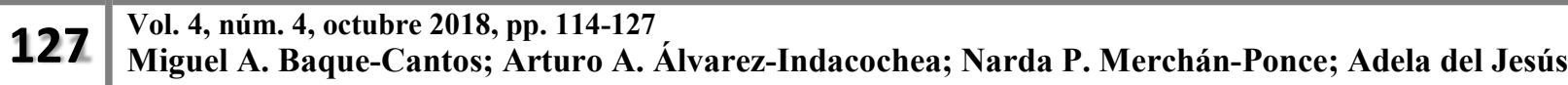
Lucio-Pillasagua
} 